\title{
El efecto del poder de mercado sobre la inversión en generación en mercados eléctricos liberalizados ${ }^{1}$
}

\author{
Fidel Castro-Rodríguez \\ Dpto. Fundamentos de Análisis Económico y RGEA, \\ Universidad de Vigo \\ Georges Siotis \\ Dpto. Economía, Universidad Carlos III de Madrid, \\ European Commission y CEPR
}

\begin{abstract}
Resumen
En este trabajo se realiza una simulación con datos del sistema eléctrico español para cuantificar el alcance del efecto directo de poder de mercado sobre la inversión en nueva capacidad de generación. Además, se describe el mecanismo regulatorio de incentivo a la inversión diseñado por las autoridades españolas y se analiza su influencia sobre las decisiones de inversión de las empresas eléctricas. Las simulaciones muestran que los niveles de capacidad instalada en un marco descentralizado con empresas con poder de mercado son significativamente inferiores a los necesarios para garantizar la cobertura de la demanda. Además, se muestra que el mecanismo de incentivos a la inversión propuesto por las autoridades españolas resulta ineficaz y altamente costoso.
\end{abstract}

Palabras Clave: Electricidad; Inversión en generación; Regulación; Liberalización.

Clasificación JEL: L13, L43, L94.

\begin{abstract}
This paper creates a simulation with data from the Spanish electricity system to quantify the effect of market investment power on new capacity of generation. It also describes the Spanish regulatory mechanism used to incentivate new investment and analyses its influence on the electricity companies' investment decisions. Simulations show that in decentralized electricity systems were firms have market power, the level of capacity installed is significantly below the level required to cover the maximum demand. Moreover, the investment incentive mechanism suggested by the Spanish government proves ineffective and highly costly.
\end{abstract}

Keywords: Electricity; Investment in generation; Regulation; Liberalization.

JEL Classification: L13, L43, L94.

${ }^{1}$ Los autores agradecen la financiación recibida del Ministerio de Educación e Innovación a través del proyecto ECO2008-05771 (Castro-Rodríguez) y del Ministerio de Educación y Ciencia mediante el proyecto SEJ2007-66268 (Siotis). Los resultados presentados en este trabajo fueron obtenidos antes de que Siotis se incorporara a la Comisión Europea. Las visiones expresadas son responsabilidad exclusiva de los autores y no reflejan necesariamente la propia de las instituciones a las que pertenecen. 


\section{Introducción}

La liberalización del sector eléctrico ha introducido importantes cambios en la organización de la industria respecto a la configuración tradicional de monopolio vertical regulado. Destaca especialmente, por un lado, la introducción de competencia en las actividades de generación y comercialización, manteniendo como reguladas las fases de transporte y distribución, y, por otro lado, la separación entre las actividades en competencia y las actividades bajo regulación. Paralelamente, se ha tenido que diseñar un mercado de intercambio entre productores y compradores, y se han establecido unas reglas de juego en sustitución de las viejas reglas basadas en una total intervención por parte de las autoridades públicas. El objetivo último de esta reforma es descentralizar tanto las decisiones de producción como las de inversión de forma que sean las propias empresas eléctricas las que tomen dichas decisiones bajo su riesgo y ventura en un entorno de libre mercado.

En todos los países en los que se han introducido las reformas liberalizadoras parece existir un consenso generalizado sobre los mecanismos necesarios para organizar la producción. Un mercado central de intercambio de energía donde vendedores y compradores presentan sus ofertas de venta y compra respectivamente, llamado mercado spot, complementado con otros mercados que permiten la corrección de posibles desequilibrios entre oferta y demanda, así como la contratación de servicios auxiliares, junto a la supervisión de los operadores del mercado y del sistema, parecen suficientes para garantizar el suministro eléctrico sin excesivos sobresaltos. Además, siempre existe la posibilidad de celebrar contratos bilaterales entre agentes, generalmente referenciados sobre el funcionamiento del mercado spot, con el objeto de minimizar riesgo e incertidumbre.

Menos unanimidad existe, sin embargo, respecto al procedimiento que debe ser utilizado para promover la inversión en nueva capacidad de generación en el nuevo escenario de libre competencia. En algunos países se ha optado por una liberalización plena y una total confianza en las señales del mercado para incentivar la nueva inversión. Bajo esta configuración la dinámica es simple: cuando aparece una clara necesidad de nueva capacidad de generación existirá una mayor tensión entre oferta y demanda que se traducirá en un incremento del precio de la energía en el mercado mayorista lo que elevará de forma significativa los beneficios del parque de generación en funcionamiento. Si no existen barreras de entrada relevantes, esos mayores beneficios atraerán nueva inversión hasta que los beneficios vuelvan a niveles normales. Por el contrario, los excesos de inversión serán penalizados con drásticas reducciones de los precios mayoristas desincentivando nuevas iniciativas inversoras, e incluso forzando el cierre o la retirada de algunas centrales de generación. A largo plazo, por tanto, el precio mayorista irá convergiendo al nivel que permita unos ingresos anuales suficientes para cubrir exactamente los costes totales de suministro.

En los sistemas eléctricos actuales, sin embargo, existen diversos factores que pueden alterar los incentivos del denominado "mercado sólo de energía" (véase 
Cramton y Stoft, 2006; Joskow, 2007, 2008; y García y Stacchetti, 2009). Por una parte, el hecho de que los activos sean altamente costosos, muy específicos y con un período de construcción de varios años introduce un nivel de incertidumbre que encarece y ralentiza las nuevas inversiones. Esto que sería preocupante en cualquier sector se convierte en especialmente peligroso en el sector eléctrico puesto que podría generar un serio problema de cobertura de la demanda dado la imposibilidad de almacenamiento de la electricidad y la alta inelasticidad de la demanda. En segundo lugar, en el sector eléctrico la entrada en funcionamiento de una nueva central no solo beneficia al propietario de dicha central y a los consumidores de la electricidad por ella producida sino también al conjunto de los consumidores del sistema que por estar conectados a una red común ven incrementada la seguridad de su suministro. Este elemento de bien público que caracteriza a la nueva inversión indica que la confianza ciega en el mercado podría conducir a un nivel de inversión inferior al socialmente eficiente. El primero de los factores podría corregirse con la introducción de contratos de largo plazo entre productores y compradores tal y como existen en otros sectores de características similares (por ejemplo en el sector petrolero), lo que reduciría el riesgo de falta de cobertura de costes para los primeros, y eliminaría la perjudicial variabilidad de precios para los segundos. El segundo de los factores, sin embargo, exigiría la introducción de algún mecanismo que permitiera internalizar los beneficios sociales de red asociados a toda nueva inversión.

No obstante, existe otro factor que en algunos países se percibe como mayor amenaza para una inversión eficiente en nueva generación: el poder de mercado. Las especiales características del sector eléctrico lo hacen muy vulnerable al ejercicio de poder de mercado, lo que puede originar distorsiones en las nuevas inversiones que pueden conducir no solo a un funcionamiento ineficiente del sistema sino también a poner en peligro el equilibrio del sistema en sí mismo.

El poder de mercado puede derivar en niveles de inversión inferiores a los socialmente eficientes por dos vías. Por un lado, de forma directa porque conociendo la relación entre capacidad instalada y funcionamiento del mercado mayorista, las empresas pueden subir los precios de la electricidad utilizando estratégicamente sus decisiones de inversión. Por otro lado, de forma indirecta porque los reguladores, conscientes del riesgo de ejercicio de poder de mercado, pueden imponer precios máximos para impedir un incremento excesivo de los precios en el mercado mayorista. Pero de esta forma estarían impidiendo que los precios suban lo suficiente como para atraer nueva inversión cuando esta sea necesaria (es lo que Cramton y Stoft (2006) denominan "missing money problem").

Este trabajo se concentra en estudiar el efecto directo del poder de mercado analizando las consecuencias que provoca el comportamiento estratégico de las empresas eléctricas en sus decisiones de inversión sobre el nivel de capacidad instalada y sobre los precios del mercado mayorista. En particular, se realiza una simulación con datos del sistema eléctrico español para cuantificar el alcance del efecto directo de poder de mercado sobre la inversión en nueva capacidad de generación. Además, se describe el mecanismo regulatorio de incentivo a la inversión diseñado por las auto- 
ridades españolas actualmente en vigor y se analiza su influencia sobre las decisiones de inversión de las empresas eléctricas.

Para realizar este análisis se plantea un modelo teórico estilizado que permite recoger adecuadamente el efecto directo de poder de mercado ${ }^{2}$. En concreto, se considera un número finito de empresas, y se representan sus decisiones como un juego en dos etapas. Durante la primera etapa, las empresas deciden el nivel de capacidad a instalar, decisión de largo plazo. En la segunda, etapa las empresas fijan precios para la energía producida. Para esta segunda etapa se estudian dos alternativas. En la primera, se supone que las empresas ofertan la energía de forma competitiva, es decir, se supone que las empresas fijan pujas iguales a sus costes marginales. En la segunda alternativa, se considera que las empresas fijan pujas con un margen constante sobre el coste marginal. De esta forma, se pretende mostrar los efectos que tiene la posibilidad de ejercer poder de mercado en el mercado mayorista sobre las decisiones de inversión.

Este modelo es utilizado para simular los efectos de la liberalización sobre el nivel de capacidad instalada, y para evaluar la influencia sobre la inversión en centrales de generación de mecanismos de remuneración de capacidad basados en el precio. Para ello se utilizan datos del sistema eléctrico español, y se simula el mecanismo de pagos de capacidad actualmente en funcionamiento.

Las simulaciones muestran que los niveles de inversión derivados del marco descentralizado con empresas eléctricas con poder de mercado son significativamente inferiores que los necesarios para garantizar la cobertura de la demanda máxima. Además, se demuestra que el mecanismo de incentivos a la inversión propuesto para el sistema eléctrico español resulta ineficaz y altamente costoso.

El trabajo se ha estructurado de la siguiente forma. En la segunda sección se describen los factores que condicionan las decisiones de inversión en nueva generación en un marco liberalizado, prestando especial atención a la influencia del poder de mercado en dichas decisiones. En la tercera sección se presenta un modelo simple que caracteriza a la industria eléctrica. La cuarta sección utiliza el modelo teórico para simular niveles de inversión en un escenario descentralizado usando datos del sector eléctrico español. La sección quinta analiza los efectos del mecanismo español de incentivo a nueva inversión, y se caracteriza el mecanismo óptimo. Por último, la sexta sección presenta las conclusiones.

\section{Factores que condicionan la nueva inversión en generación de electricidad}

La reforma liberalizadora del sector eléctrico surgida para promover un uso más eficiente de los recursos ha llevado a una importante reducción de los márgenes de capacidad de reserva en todos los países de la OCDE (Auriol and Biancini,

${ }^{2}$ Se utiliza una versión simplificada del modelo teórico desarrollado en Castro-Rodríguez et al., 2009. 
2009). Al mismo tiempo, se ha incrementado el número de casos de fallos de suministro en alguno de los sistemas liberalizados como California, Nueva York, Italia, Noruega, Suecia, Brasil, Argentina, Chile, Nueva Zelanda y España (véanse De Vries, 2006; Fabra, 2007; Joskow, 2007; y Neuhoff y De Vries, 2004). Todo ello ha generado una enorme preocupación y un gran debate sobre si los mercados pueden proporcionar los incentivos suficientes para una inversión adecuada y a tiempo que permita garantizar el suministro de electricidad a precios razonables (ver García y Stacchetti, 2009) $)^{3}$.

El elemento principal del proceso de reforma ha sido la sustitución de la planificación centralizada en la que se basaba el modelo tradicional de monopolios públicos integrados verticalmente por un mecanismo descentralizado de decisiones realizadas a través del mercado. En el nuevo marco liberalizado ya no existen planes de expansión de capacidad ni procedimientos de explotación unificada sino que son las empresas eléctricas las que tienen que decidir si desarrollar o no un proyecto de inversión en función de su expectativa de beneficios. La cuestión importante bajo este nuevo paradigma es si las fuerzas del mercado son suficientes para impulsar decisiones eficientes. Algunos países confían plenamente en el mercado, otros buscan soluciones complementarias, unos pocos recelan completamente del mercado e intervienen directamente. Pero, ¿cuáles son los factores que hacen diferente el problema de inversión en nueva capacidad en el sector eléctrico?

En primer lugar, el funcionamiento de los mercados eléctricos está sometido a una gran incertidumbre. Los activos eléctricos son muy caros, muy específicos y se necesita un largo tiempo para la construcción de centrales eléctricas. La gran volatilidad de precios debido a la alta variabilidad de la demanda (muy condicionada por factores meteorológicos) acrecienta la incertidumbre y el riesgo de inversión. Aunque este es un factor presente en otras industrias donde se producen grandes inversiones en un entorno de fuerte variabilidad de precios (por ejemplo, petróleo o gas natural), el escaso desarrollo de instrumentos de cobertura de riesgo en el mercado eléctrico como la contratación a plazo, la contratación bilateral o los mercados de futuros, aconsejan que, al menos en una primera etapa, se introduzca algún mecanismo que complemente al mercado y reduzca la incertidumbre que puede poner en peligro la seguridad de suministro de un servicio esencial para la sociedad.

En segundo lugar, los operadores de los sistemas eléctricos no confían en el mercado para resolver situaciones de desequilibrio debido a que tanto la demanda como la oferta son demasiado inelásticas para reaccionar de forma rápida. Además, no se pueden utilizar inventarios dada la imposibilidad de almacenar electricidad. Por ello, para evitar los altos costes asociados a una interrupción de suministro, y mayores costes aún, si se produce un colapso del sistema, lo que no es aceptable ni técnica ni

${ }^{3}$ En los últimos años, como consecuencia de la crisis económica mundial, se ha producido un importante descenso de la demanda de electricidad que ha suavizado el problema de falta de suministro. Adicionalmente, en algunos sistemas eléctricos como el español los incentivos públicos a las energías renovables han promovido incrementos significativos de inversión en nueva capacidad que han llevado a fuertes aumentos de los márgenes de reserva (véase el informe CNE, 2009). 
políticamente, los operadores prefieren utilizar procedimientos ajenos al mercado como el racionamiento aleatorio de la demanda. Pero la intervención de los operadores "por fuera del mercado" distorsiona las señales de precios lo que conduce a decisiones no eficientes por parte de los agentes: los productores no invierten ni producen adecuadamente, y los consumidores no usan el servicio eficientemente. Quizás sea necesario algún tiempo de maduración para que se tenga plena confianza en el mercado, pero en cualquier caso se deben ir planteando políticas que hagan mas flexibles tanto a la oferta como a la demanda. Mientras tanto, es necesario el uso de mecanismos complementarios del mercado que permitan mantener el equilibrio del sistema pero que interfieran lo menos posible en el funcionamiento el mismo.

En tercer lugar, la inversión en una nueva central de generación posee características de bien público, dado que la construcción de una nueva central de generación no solo beneficia a su propietario y a los consumidores que consumen directamente su producción, sino también al resto de agentes conectados a la red que ven incrementada la seguridad de suministro por reducirse la probabilidad de fallo del sistema. Como los inversores individuales sólo tendrán en cuenta los beneficios privados y no los sociales su inversión será inferior a la socialmente óptima. Será necesario, por tanto, buscar algún mecanismo que internalize la externalidad, y que distorsione lo menos posible las decisiones de los agentes económicos.

En cuarto lugar, en los sistemas eléctricos liberalizados existe un gran poder de mercado en manos de las empresas eléctricas ${ }^{4}$. Este poder de mercado influye en las decisiones de inversión por dos vías. La primera, que podemos llamar directa, se deriva de la relación inmediata que existe entre el nivel de capacidad instalada y los precios del mercado mayorista. En un mercado en donde el precio horario resulta de la intersección entre demanda y oferta cuanto mayor sea el número de horas en las que exista una oferta restringida mayor será el precio medio del mercado mayorista. Por ello, las empresas decidirán estratégicamente el nivel de inversión buscando el máximo beneficio teniendo en cuenta esa relación entre capacidad instalada del sistema y precio (véase García y Stacchetti, 2009). La segunda, que podemos denominar indirecta, es consecuencia de que los reguladores no confían en el comportamiento de las empresas, y por ello, introducen instrumentos y políticas para impedir o mitigar el ejercicio de poder de mercado. Así, por ejemplo, es frecuente que los reguladores impongan un límite máximo al precio del mercado mayorista para impedir que en periodos de fuerte restricción de capacidad el precio pueda alcanzar niveles muy altos. Esta práctica claramente interfiere en el funcionamiento del mercado, distorsionando una vez más las señales de precios y, con ello, las decisiones

${ }^{4}$ El mercado eléctrico presenta una serie de características que pueden facilitar la aparición de poder de mercado: la imposibilidad o alto coste de almacenamiento de la electricidad que impide la utilización de inventarios como factor de moderación de los precios, la baja elasticidad de la demanda y la oferta que apenas responden a fuertes incrementos del precio, y el alto nivel de concentración de la estructura empresarial que posibilita la concertación de prácticas colusivas. Adicionalmente, las restricciones de la red de transporte pueden originar monopolios locales que permitan el abuso de determinados generadores. 
de inversión de las empresas. Será, pues, recomendable la introducción de medidas que reduzcan tanto el poder de mercado como el riesgo de ejercicio de ese poder de mercado, afectando tanto a las estructura de mercado como al comportamiento de las empresas.

Mientras que en algunos países se ha confiado plenamente en las decisiones de inversión que han surgido del mercado, en otros se han implementado diferentes mecanismos para corregir las posibles imperfecciones mencionadas anteriormente. En general, podemos agrupar a estos mecanismos en dos categorías: los basados en el precio, en los que el regulador fija el precio de la capacidad y los agentes privados deciden los niveles de inversión; y los basados en la cantidad, en los que el regulador decide el nivel necesario de capacidad y su precio es determinado por mecanismos de mercado. En el mercado eléctrico español se ha optado tanto en el primer mecanismo ("garantía de potencia") como en el actualmente vigente ("incentivo a la inversión") por un instrumento de incentivo a la nueva inversión basado en el precio.

En este trabajo se evalúa el efecto directo del poder de mercado sobre las decisiones de inversión por parte de empresas eléctricas en un marco completamente desregulado. Asimismo, se analiza la influencia del mecanismo de incentivo a la inversión utilizado actualmente en el sistema eléctrico español.

\section{Comportamiento estratégico en las decisiones privadas de inversión: principales características del modelo teórico}

En este trabajo seguimos el modelo de Castro-Rodríguez et al. (2009) planteado para simular los efectos del poder de mercado sobre la inversión en generación. Este modelo recoge de forma estilizada la interacción entre agentes en un mercado eléctrico horario, suponiendo que no existe intervención, o que esta intervención es mínima. En particular, se supone que cuando se producen fuertes restricciones de capacidad el regulador permite subir los precios. Tampoco se contempla la posibilidad de que cuando los precios sean altos entren tecnologías alternativas como la autogeneración. Aunque los resultados que se presentan en las simulaciones ofrecen una orientación del problema, no reflejan lo que probablemente esté ocurriendo en el mercado real. La información que proporcionan permite una mejor comprensión del funcionamiento del mercado, y sirve de base para la adopción de medidas que mejoren las decisiones de inversión en mercados eléctricos liberalizados.

Se distinguen dos tipos de demanda horaria. El primer tipo representa la demanda de electricidad que puede ser ajustada dentro de la hora tal como la de las centrales de bombeo o la de los consumidores con sofisticados instrumentos de medición que pueden modificar su consumo en función del precio en tiempo real (demanda modulable). En la práctica esta demanda representa una pequeña proporción de la demanda total (entre 1 y un 5\%). El segundo tipo representa la parte de la demanda que no puede ser ajustada dentro de una hora bien porque los consumidores no reciben información sobre el precio horario o bien porque las condiciones tecnológicas 
no permiten hacerlo, tal es el caso de los consumidores que pagan una tarifa fija por KWh con independencia del precio horario (demanda no modulable). Este grupo es totalmente insensible al precio horario. Además, la demanda horaria presenta una alta variabilidad debido tanto a fluctuaciones en la actividad económica como a cambios en las condiciones meteorológicas.

Respecto a la oferta, por simplicidad se considera que existe una sola tecnología con coste marginal constante de tal forma que la curva de oferta agregada corresponde con una línea horizontal a la altura del coste marginal hasta el nivel de capacidad instalada total. Esto implica que una vez alcanzado el nivel de capacidad disponible, el coste marginal se hace infinito reflejando el hecho de que la oferta no puede ser expandida a corto plazo $^{5}$. Debido a las tareas de mantenimiento y a fallos imprevistos del equipo generador, solo una fracción del parque instalado está disponible en cada momento del tiempo.

El precio horario es determinado por la intersección entre la demanda y la ofer$t^{6}{ }^{6}$. A medida que la oferta se hace más restringida el precio horario se va incrementando con lo que parte de la demanda sensible al precio se va reduciendo. Cuando la capacidad disponible sea tal que ya toda la demanda modulable se ha retirado y no sea posible abastecer a toda la demanda no modulable, el operador del sistema cortará el suministro aleatoriamente a algunos consumidores a tarifa para reestablecer el equilibrio del sistema, y fijará un precio máximo igual a la valoración media que conceden dichos consumidores a un $\mathrm{kWh}^{7}$.

Para calcular el coste fijo unitario asociado con una unidad de nueva capacidad, se supone una vida útil fija para cada unidad generadora y se distribuyen los costes fijos entre los kWh que se esperan producir, lo cuál dependerá de la capacidad agregada. Claramente, el nivel de utilización de una planta eléctrica está inversamente relacionado con la capacidad agregada dado que cuanto mayor sea la capacidad instalada menor será la probabilidad con la que una planta será despachada.

Para derivar el nivel de inversión correspondiente a una situación en la que las empresas son completamente libres para elegir la capacidad a instalar, se considera un número finito de empresas, y se representan sus decisiones como un juego en dos etapas. Durante la primera etapa, las empresas deciden el nivel de capacidad a instalar, decisión de largo plazo. En la segunda etapa, las empresas deciden el precio para su energía. En esta segunda etapa se estudian dos alternativas. En la primera, se supone que las empresas ofertan la energía de forma competitiva, es decir, se supone que las

${ }^{5}$ Se han hecho simulaciones considerando una curva de oferta creciente reflejando un parque de generación con múltiples tecnologías y los resultados no han diferido cualitativamente. La razón es que los beneficios obtenidos por las unidades inframarginales (que no fijan precio) son significativamente más pequeños que los incrementos de beneficios derivados de un precio mayorista más alto.

${ }^{6}$ Esto equivale a decir que el precio horario con el que se remunera la energía producida viene determinado por la central de coste más alto que es despachada, o también conocido como subasta de precio uniforme.

${ }^{7}$ Esto es lo que la literatura denomina "valor de la energía no suministrada", o "value of lost load" (VOLL) en su acepción inglesa. 
empresas fijan pujas iguales a sus costes marginales (márgenes nulos) ${ }^{8}$. Dado que los agentes no actúan estratégicamente en la segunda etapa del juego, este modelo es cercano, aunque no equivalente, a un juego en una sola etapa en el que los agentes tienen que decidir de forma simultánea sobre capacidad y producción. La diferencia surge de la existencia de incertidumbre: como existirá exceso de capacidad cuando la demanda sea baja, y las empresas no pueden obtener rentas por fijar precios iguales a costes marginales, las decisiones de inversión serán alteradas en la primera etapa del juego. En particular, las empresas están interesadas en reducir la inversión en la primera etapa para poder alcanzar un precio medio superior en el mercado horario al existir un mayor número de horas en los que la capacidad instalada esté restringida. Es decir, aunque las empresas están interesadas en invertir en nueva capacidad para ampliar su cuota de mercado, son conscientes que una mayor inversión deprimirá los precios y sus beneficios, por lo que al final tratarán de mantener un nivel de capacidad que esté restringido el mayor tiempo posible. Este resultado está en línea con el obtenido en literatura (véase Grimm y Zoettl, 2006, García y Stacchetti, 2009). Claramente, con este supuesto se está aproximando el caso extremo de ejercicio de poder de mercado en la etapa de inversión, dado que las empresas no cuentan con la posibilidad de manipular precios en el mercado de producción. En la segunda alternativa, se considera que las empresas fijan pujas con un margen constante sobre el coste marginal. De esta forma, se pretende mostrar los efectos que tiene la posibilidad de ejercer poder de mercado en el mercado mayorista sobre las decisiones de inversión.

En este modelo, el beneficio que obtiene cada empresa no sólo depende de su nivel de inversión sino también de la inversión realizada por otras empresas.

\section{Simulación de los efectos de poder de mercado sobre la inversión}

En este apartado se presenta la resolución numérica del modelo teórico utilizando datos del mercado eléctrico español. Para ello, en primer lugar se describen los datos utilizados y posteriormente se detallan los resultados.

\subsection{Datos}

Para aproximar la variabilidad de la demanda se utiliza la distribución de demanda horaria para 2005, información recibida del operador del mercado español (OMEL). La demanda horaria ha sido proyectada hasta 2010 utilizando previsiones de crecimiento de demanda suministradas por el operador del sistema (REE). Nues-

${ }^{8}$ Bajo este marco se está suponiendo que las empresas no pueden ejercitar poder de mercado en el mercado spot. Aunque esto podría calificarse como poco realista, se introduce para cuantificar el máximo efecto directo del poder de mercado sobre la inversión en capacidad. La relajación de este supuesto podría suponer una menor intensidad del efecto del poder de mercado sobre la inversión. 
tras simulaciones se centran en el año 2008. Para un análisis detallado de la construcción de la función de distribución de la demanda utilizada puede verse CastroRodríguez et al. (2009).

Dadas las particulares características de funcionamiento de las fuentes renovables, se ha detraído de la demanda total la parte cubierta con energía renovable, por lo que la demanda utilizada en las simulaciones corresponde con la parte de la demanda cubierta exclusivamente con tecnología no renovable (demanda térmica).

Como aproximación de la valoración media que los consumidores sujetos a tarifa conceden a la energía no suministrada (VOLL) se ha utilizado el ratio del PIB por MWh para el año 1999, obteniendo una cifra de 3.005,5 Euros.

Con respecto a la demanda, se considera que la pendiente de la misma se mantiene constante a lo largo del año, aunque varíe su valor máximo en cada hora, y que el porcentaje de demanda sensible al precio es del 5\% de la demanda máxima, lo que implica que el restante $95 \%$ corresponde fundamentalmente con demanda a tarifa fija, o demanda que no puede variar horariamente.

Se considera que toda la nueva inversión se realiza en centrales de ciclo combinado para las que suponemos un coste variable de 47 Euros/MWh ${ }^{10}$.

Para aproximar el coste unitario de capacidad se han dividido los costes fijos totales de inversión de una planta de generación entre los MWh que se espera que produzca dicha planta a lo largo de su vida útil. Esto significa que el coste unitario de capacidad es más bajo cuanto mayor es el número de horas que es despachada la planta. Por ejemplo, si se supone un nivel medio de utilización de 7.500 horas al año para una planta de ciclo combinado de $395 \mathrm{MW}$ con una vida útil de 20 años, su producción esperada será de $58.065 \mathrm{GWh}$, y el coste unitario de capacidad resultante será 6,53 Euros/MW.

Para cada planta de generación se considera un ratio medio de disponibilidad del $92,5 \%$, en base a la información del operador del sistema.

Con respecto al número de empresas activas en el mercado, se presentan simulaciones relativas a tres escenarios. El primer escenario corresponde a una situación de libre entrada. Es decir, aparte de problemas de divisibilidad (se considera que el tamaño mínimo de una planta de generación de ciclo combinado no puede ser inferior a 387,1 MW), el número máximo de empresas activas queda determinado por la necesidad de cubrir costes fijos (condición de beneficio no negativo) y por la restricción de tamaño mínimo. Combinando estas restricciones obtenemos un número de 70 empresas. Claramente, este escenario es muy poco probable en la práctica dadas las importantes restricciones de entrada derivadas de la limitación de localizaciones de emplazamiento o de las restricciones impuestas por la red de transporte.

${ }^{9}$ Se ha adoptado el supuesto de alisamiento de la curva de carga, en virtud del cuál la producción hidroeléctrica es distribuida para cubrir las horas de demanda punta hasta que toda la capacidad hidráulica se haya agotado. Para ello, se han tenido en cuenta las restricciones técnicas que marcan el máximo y mínimo de producción hidroeléctrica que puede ser despachada en una hora.

${ }^{10}$ Se han analizado variaciones en todos los parámetros relevantes sin obtener cambios significativos en los resultados. 
El segundo escenario representa un mercado eléctrico bastante activo con hasta 20 empresas. El tercer escenario corresponde con un escenario claramente restrictivo, en lo que a competencia se refiere en el que solamente operan 6 empresas. Este escenario podría replicar adecuadamente el número de empresas actualmente en operación. En el cuadro 1 se presentan los valores de todos los parámetros utilizados en las simulaciones.

\section{CUADRO 1}

VALORES DE LOS PARÁMETROS

\begin{tabular}{|l|c|}
\hline \multicolumn{1}{|c|}{ Parámetro } & Valor del escenario base \\
\hline Distribución de demanda térmica & \\
\hline Mínima (GWh) & 5.585 \\
\hline Máxima (GWh) & 31.383 \\
\hline Media (GWh) & 20.616 \\
\hline Desviación típica $(\mathrm{GWh})$ & 4.622 \\
\hline Valoración medio de un MWh (€/MWh) & 3.005 \\
\hline Características de una planta de CC & \\
\hline Tamaño mínimo (MW) & 387,1 \\
\hline Coste variable (€/MWh) & 47 \\
\hline Coste fijo (millones de Euros) & 632 \\
\hline Vida útil (años) & 20 \\
\hline Ratio de disponibilidad & $92,5 \%$ \\
\hline Número de empresas & $6,20,70$ \\
\hline
\end{tabular}

\section{Resultados}

En el cuadro 2 se presentan los resultados de capacidad disponible como porcentaje de la demanda máxima para cada uno de los escenarios analizados. Como se observa, para cualquiera de los escenarios analizados la libre elección de capacidad por parte de las empresas conduce a un nivel de capacidad disponible insuficiente para cubrir la demanda máxima, por lo que existirán algunas horas en las que el operador del sistema deberá cortar de manera aleatoria el suministro de electricidad a algunos consumidores. La falta de cobertura y, por tanto, la cantidad de energía no suminis- 
trada será mayor cuanto menor sea el número de empresas operando en el mercado, reflejando el resultado clásico de la teoría de oligopolio de que la reducción del número de empresas facilita el ejercicio de poder de mercado, en este caso a través de una mayor reducción de la capacidad instalada.

\section{CUADRO 2}

CAPACIDAD DISPONIBLE COMO PORCENTAJE DE DEMANDA MÁXIMA Y DEL NIVEL DE CAPACIDAD RECOMENDADO POR LOS OPERADORES DEL SISTEMA ELÉCTRICO (1,1 VECES LA DEMANDA MÁXIMA)

\begin{tabular}{|c|c|c|}
\hline No empresas & $\begin{array}{c}\text { Capacidad Disponible } \\
\text { como porcentaje } \\
\text { sobre demanda máxima }\end{array}$ & $\begin{array}{c}\text { Capacidad Disponible } \\
\text { como porcentaje } \\
\text { sobre 1,1 veces la demanda máxima }\end{array}$ \\
\hline \multicolumn{3}{|c|}{ Precios iguales a costes marginales } \\
\hline 6 & $72 \%$ & $62 \%$ \\
\hline 20 & $90 \%$ & $82 \%$ \\
\hline 70 & $94 \%$ & $85 \%$ \\
\hline \multicolumn{2}{|c|}{ Precios con un margen del 15\% sobre los costes marginales } \\
\hline 6 & $72 \%$ & $62 \%$ \\
\hline 20 & $90 \%$ & $82 \%$ \\
\hline 70 & $94 \%$ & $85 \%$ \\
\hline
\end{tabular}

Teniendo en cuenta que la mayoría de los operadores de sistemas eléctricos consideran razonable para garantizar el suministro un margen de cobertura del $10 \%$ sobre la demanda máxima, en el cuadro 2 también se recogen los datos de capacidad disponible como porcentaje de ese nivel de capacidad recomendado. Bajo esta premisa la falta de capacidad se hace mas notoria llegando casi al $40 \%$ para el escenario de mínima competencia en el que solamente 6 empresas ofertan electricidad.

En el cuadro 2 se presenta también la capacidad disponible como porcentaje de la demanda máxima y de la capacidad recomendada por los operadores de sistemas eléctricos, bajo la alternativa de que las empresas hacen sus pujas de oferta con un margen del 15\% sobre el coste marginal. Como puede observarse, la posibilidad de ejercitar poder de mercado en el mercado mayorista no afecta a los niveles de inversión privados, posiblemente porque los márgenes que se consiguen en el mercado mayorista no compensan la reducción de precios provocada por el aumento de la capacidad disponible. En definitiva, este resultado muestra que serían necesarios importantes márgenes de beneficio en el mercado mayorista para lograr aumentos signifi- 
cativos de capacidad disponible. En cualquier caso, aun sin tener en cuenta el hecho de que en este escenario los aumentos de inversión proceden de una fuerte elevación de precios del mercado mayorista - con el consiguiente perjuicio para los consumidores-, no hay garantía de que se elimine la falta de cobertura en algunas horas del año.

Introducción de un precio máximo en el mercado mayorista

En el cuadro 3 se presenta la capacidad disponible bajo los mismos escenarios del cuadro anterior pero esta vez considerando un precio máximo en el mercado mayorista por debajo del "valor de energía no suministrada". Los resultados muestran que la introducción de un precio máximo reduce el nivel de capacidad instalado por las empresas eléctricas. La razón es que al existir un precio máximo las empresas no pueden recibir los grandes beneficios derivados de los altos precios en horas de capacidad restringida. Por ello, reducen el nivel de inversión para tener un mayor número de horas con capacidad restringida.

\section{CUADRO 3}

CAPACIDAD DISPONIBLE COMO PORCENTAJE DE DEMANDA MÁXIMA

Y DEL NIVEL DE CAPACIDAD RECOMENDADO POR LOS OPERADORES DEL SISTEMA ELÉCTRICO (1,1 VECES LA DEMANDA MÁXIMA) CUANDO EXISTE UN PRECIO MÁXIMO DE 180 €/MWH

\begin{tabular}{|c|c|c|}
\hline N empresas & $\begin{array}{c}\text { Capacidad Disponible } \\
\text { como porcentaje } \\
\text { sobre demanda máxima }\end{array}$ & $\begin{array}{c}\text { Capacidad Disponible } \\
\text { como porcentaje } \\
\text { sobre 1,1 veces la demanda máxima }\end{array}$ \\
\hline \multicolumn{3}{|c|}{ Precios iguales a costes marginales } \\
\hline 6 & $66 \%$ & $60 \%$ \\
\hline 20 & $75 \%$ & $68 \%$ \\
\hline 70 & $79 \%$ & $71 \%$ \\
\hline & Precios con un margen del 15\% sobre los costes marginales \\
\hline 6 & $67 \%$ & $61 \%$ \\
\hline 20 & $75 \%$ & $69 \%$ \\
\hline 70 & $79 \%$ & $71 \%$ \\
\hline
\end{tabular}

Los resultados anteriores se confirman y entienden mejor si observamos los precios medios del mercado bajo los distintos escenarios. El cuadro 4 presenta los precios medios cuando existe un precio máximo de $180 € / \mathrm{MWh}$ considerando las 
dos alternativas de competencia en la segunda etapa: precio igual a coste marginal y precio con un margen del $15 \%$ sobre el coste marginal ${ }^{11}$. Como puede observarse, el precio medio crece significativamente a medida que se reduce el número de empresas, y aunque también crece cuando se aplica un margen sobre el coste marginal este crecimiento no es muy significativo.

\section{CUADRO 4}

\section{PRECIOS MEDIOS DEL MERCADO MAYORISTA}

\begin{tabular}{|c|c|c|c|}
\hline \multicolumn{4}{|c|}{ Precio máximo de $180 € / M W h$ y precios iguales a costes marginales } \\
\hline & $\begin{array}{l}\text { Térmico } \\
(€ / \mathrm{MWh})\end{array}$ & $\begin{array}{l}\text { Hidráulico } \\
(€ / \mathrm{MWh})\end{array}$ & $\begin{array}{c}\text { Total } \\
(€ / \mathrm{MWh})\end{array}$ \\
\hline 6 & 102 & 130 & 106 \\
\hline 20 & 76 & 91 & 77 \\
\hline 70 & 70 & 82 & 71 \\
\hline \multicolumn{4}{|c|}{ Precio máximo de $180 € / \mathrm{MWh}$ y precios con un margen del $15 \%$ sobre costes mar } \\
\hline & $\begin{array}{l}\text { Térmico } \\
(€ / \mathrm{MWh})\end{array}$ & $\begin{array}{l}\text { Hidráulico } \\
\text { (€/MWh) }\end{array}$ & $\begin{array}{c}\text { Total } \\
(€ / \mathrm{MWh})\end{array}$ \\
\hline 6 & 105 & 129 & 108 \\
\hline 20 & 81 & 95 & 82 \\
\hline 70 & 75 & 87 & 77 \\
\hline
\end{tabular}

\section{Análisis del mecanismo de incentivos a la inversión del sistema eléctrico español}

Desde el inicio del proceso liberalizador, en el sector eléctrico español siempre ha estado vigente un mecanismo de remuneración de capacidad como incentivo a nueva inversión. Inicialmente existía el concepto "garantía de potencia" en virtud del cuál se remuneraba a las plantas de producción acorde a su potencia disponible

${ }^{11}$ El precio medio total se obtiene como suma ponderada del precio medio recibido por la energía producida con fuentes renovables y del precio medio recibido por la energía producida con la capacidad térmica. En particular, el precio medio total es calculado asignado una ponderación del 88,5\% al precio medio térmico y del 11,5\% el precio medio hidráulico, en consonancia con la producción de ambos tipos de tecnologías. 
declarada. Con la Orden ITC/2794/2007 se sustituye el anterior mecanismo por uno nuevo denominado "pagos por capacidad" que incluye dos tipos de conceptos. En primer lugar, un incentivo a la disponibilidad a corto y medio plazo para aquellas centrales que, con mayor probabilidad, pudieran no resultar programadas cuando más las necesita el sistema (térmicas de fuel e hidráulica regulable). En segundo lugar, un incentivo a la inversión destinado exclusivamente a promover la construcción y puesta en servicio de nuevas instalaciones de generación. En esta sección describimos el mecanismo de incentivo a la inversión y simulamos sus efectos sobre las decisiones de las empresas.

\section{Descripción del nuevo mecanismo de incentivo a la inversión}

El nuevo mecanismo de incentivo a la inversión establece una remuneración para las instalaciones con una potencia instalada igual o superior a $50 \mathrm{MW}$ que entren en funcionamiento a partir del 1 de enero de 2008. Esta remuneración, que será percibida durante un periodo de 10 años, es una función decreciente del índice de cobertura (IC), definido como el cociente entre la potencia instalada y la demanda máxima. En particular, si el IC es inferior a 1,1 percibirán al año 28.000 Euros por MW. Si el IC se sitúa entre 1,1 y 1,29 la remuneración anual por MW quedará determinada por la siguiente expresión: 193.000-150.000 x IC. A partir de un IC superior a 1,29 la remuneración será cero. El gráfico 1 muestra la evolución de la remuneración como función del IC.

\section{GRÁFICO 1}

MECANISMO ESPAÑOL INCENTIVO A LA INVERSIÓN SEGÚN ORDEN ITC/2394/2007

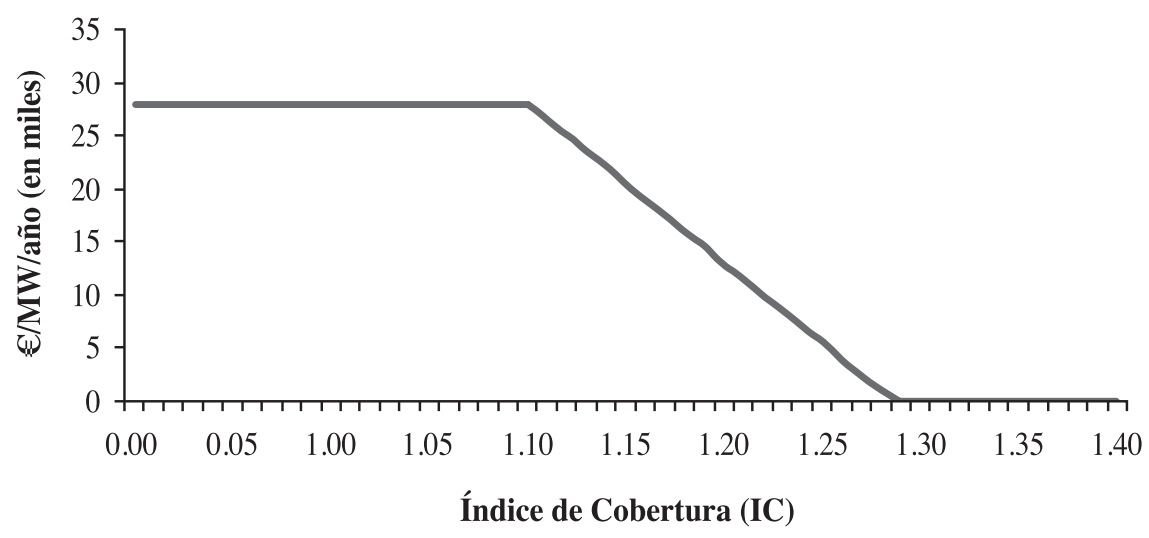


Con objeto de realizar la simulación del mecanismo la remuneración debe expresarse en $€ / M W h$ como función de la capacidad instalada (k). Teniendo en cuenta que el índice de cobertura se define como

$$
I C=\frac{k}{D \max }
$$

y que un año tiene 8.760 horas, la remuneración por hora (€/MWh) será:

$$
\begin{cases}\frac{28.000}{8.760} & \text { si } \quad k<1,1 D \max \\ \frac{\left(193.000-150.000 \frac{k}{D \max }\right)}{8.760} & \text { si } \quad k \geq 1,1 D \max \end{cases}
$$

Gráficamente puede expresarse como:

\section{GRÁFICO 2}

\section{REMUNERACIÓN A LA INVERSIÓN SEGÚN CAPACIDAD INSTALADA}

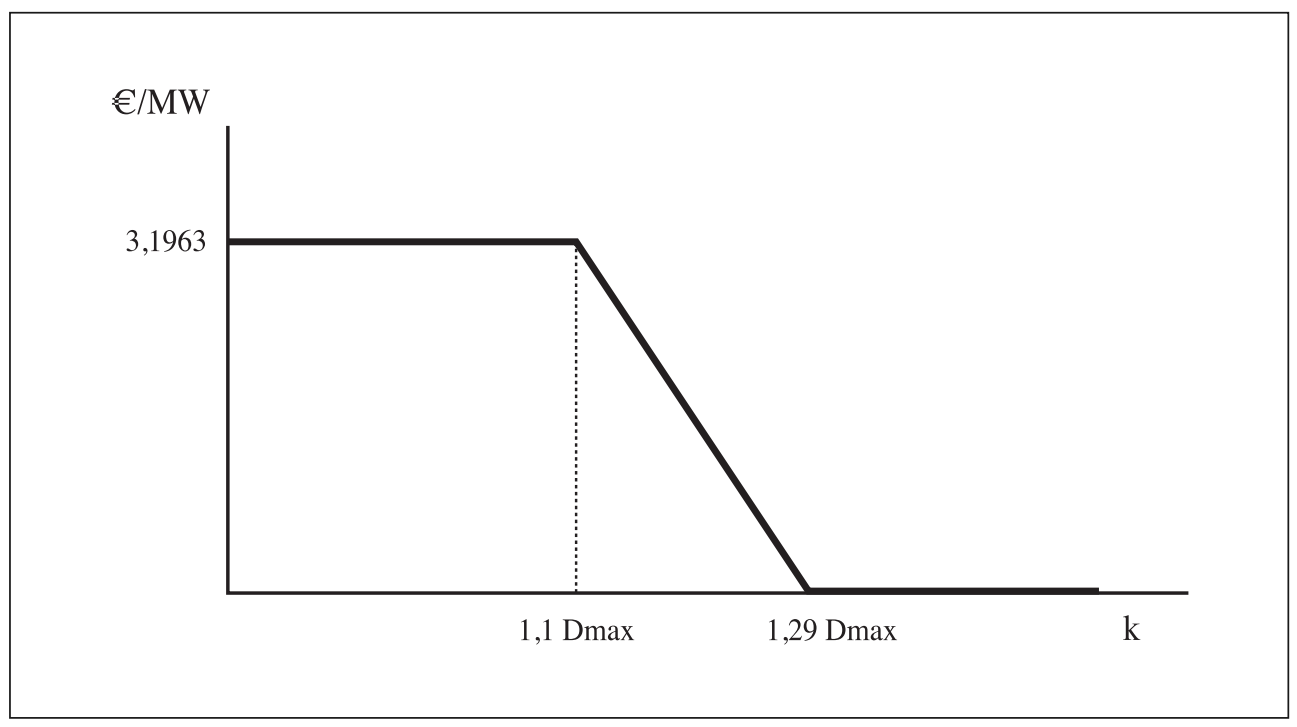

Por ejemplo, para un índice de cobertura inferior a 1,1 la remuneración por nueva inversión será de 3,1963 €/MWh. 
Efectos del mecanismo sobre la inversión en capacidad

El cuadro 5 presenta los resultados de capacidad instalada cuando se introduce el mecanismo de remuneración de nueva inversión, considerando los escenarios con y sin precio máximo en el mercado mayorista, y con precios iguales a los costes marginales y con un margen constante del $15 \%$ sobre los costes marginales, respectivamente.

\section{CUADRO 5}

CAPACIDAD INSTALADA ADICIONAL EN MW CUANDO SE INTRODUCE EL MECANISMO DE REMUNERACIÓN DE CAPACIDAD

\begin{tabular}{|c|c|c|}
\hline $\mathbf{n}$ & Sin Pmax.; $\mathbf{p}=\mathbf{C M g}$ & $\mathbf{P m a x}=\mathbf{1 8 0} ; \mathbf{p}=\mathbf{C M g}$ \\
\hline 6 & 10 & 136 \\
\hline 20 & 4 & 100 \\
\hline 70 & 2 & 81 \\
\hline $\mathbf{n}$ & Sin Pmax.; Margen 15\% & $\mathbf{P m a x}=\mathbf{1 8 0} ;$ Margen 15\% \\
\hline 6 & 51 & 636 \\
\hline 20 & 15 & 120 \\
\hline 70 & 6 & 251 \\
\hline
\end{tabular}

Puede observarse que el mecanismo tiene un efecto mínimo sobre los incentivos a la inversión. Además, su diseño parece tener efectos perversos dado que su eficacia disminuye con el grado de competencia si este es aproximado a través del número de empresas, dado un número de empresas funciona mejor cuando existe poder de mercado (margen positivo sobre coste marginal), y su eficacia también aumenta cuando se impone un precio máximo en el mercado mayorista. En definitiva, el mecanismo propuesto no incentiva los niveles de inversión que pretende el regulador con el objetivo de alcanzar una razonable garantía de suministro.

\section{Mecanismo de pago de capacidad óptimo}

A partir del modelo teórico es posible determinar cuál sería la remuneración que induciría el nivel de inversión que garantice un margen de reserva considerado apropiado por el regulador. Para ello, partiendo de un número dado de empresas (la si- 
mulación está hecha para 70 empresas) calculamos el pago necesario para incentivar una inversión que permita alcanzar el nivel de cobertura deseado por el regulador. En este ejercicio se plantea como objetivo alcanzar un margen de cobertura del 10\% sobre la demanda máxima ( $\mathrm{k}=1,1 \mathrm{Dmax})$. El gráfico 3 presenta el mecanismo de remuneración óptimo en un escenario en el compiten 70 empresas, no existe límite máximo al precio del mercado mayorista y las empresas actúan competitivamente en el mercado spot fijando precios iguales a los costes marginales.

\section{GRÁFICO 3}

MECANISMO ÓPTIMO DE PAGO POR CAPACIDAD PARA ALCANZAR UN MARGEN DE RESERVA DEL 10\% SOBRE LA DEMANDA MÁXIMA

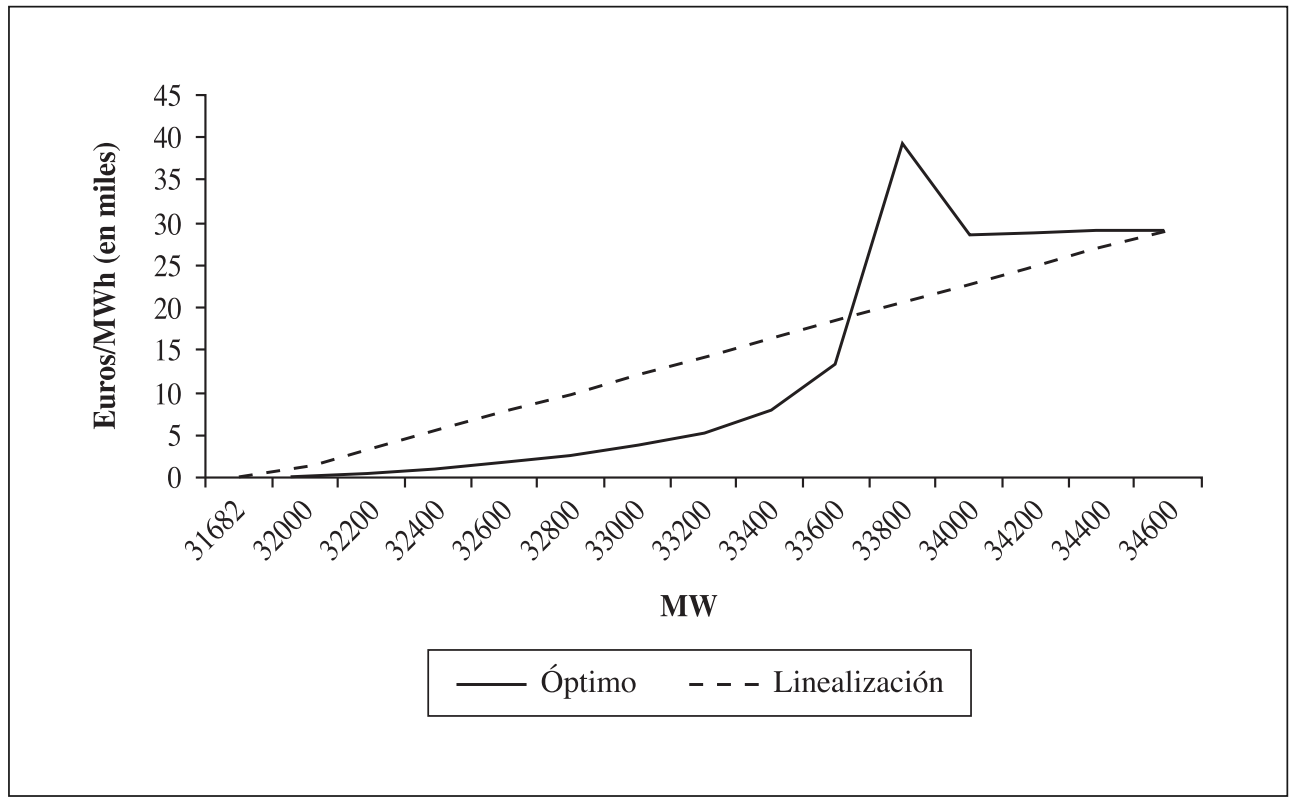

El mecanismo óptimo tiene un perfil que viene determinado por la evolución del beneficio marginal de cada empresa a medida que se va incrementando la capacidad instalada. Con cada unidad adicional de capacidad la empresa debe afrontar no sólo el coste marginal de producción (en caso de que la nueva unidad llegue a ser despachada) sino también el coste fijo de la nueva capacidad. Por su parte, su ingreso marginal se incrementa como consecuencia de que aumenta sus ventas aunque en menor proporción que el coste marginal. A partir de un determinado nivel de capacidad instalada el ingreso marginal se reduce como consecuencia de la disminución del precio medio mayorista debido a la reducción de horas en las que la capacidad está restringida. Todo ello hace que el beneficio marginal (ingreso marginal menos coste marginal total) sea cada vez más negativo a medida que crece el nivel de capacidad ins- 
talada. Entonces, el pago de capacidad necesario para incentivar nueva inversión en capacidad deberá contrarrestar la caída del beneficio marginal y por eso debe ser creciente con la capacidad instalada. El punto de inflexión que se observa en el pago se debe a que a partir de un determinado nivel de capacidad (34.000 MW), las unidades adicionales de capacidad no serán despachadas a precio igual al coste marginal por existir exceso de capacidad, por lo que el ingreso marginal se anula, aunque los costes de capacidad se mantienen. El gráfico 3 también muestra como debiera ser el pago de capacidad lineal con objeto de alcanzar el nivel de capacidad deseado, con un perfil creciente hasta la inversión deseada, claramente opuesto al postulado por el actual mecanismo español.

En definitiva, el pago por capacidad tiene que ser creciente con el nivel de capacidad puesto que una unidad adicional no solo es costosa sino que además reduce el precio pagado a todas las unidades inframarginales. Además, el coste de este mecanismo es extremadamente alto porque se necesita una transferencia monetaria a la empresa suficiente para desplazar su curva de ingreso marginal hasta que esta intercepte a la curva de coste marginal justamente para el nivel de capacidad deseado ${ }^{12}$.

\section{Conclusiones}

Los mercados eléctricos presentan condiciones que facilitan el ejercicio de poder de mercado. Por un lado, la imposibilidad de almacenamiento impide el uso de inventarios para corregir fuertes oscilaciones de los precios. Por otro lado, la baja elasticidad de la demanda derivada de la escasa sustituibilidad del producto y de la falta de exposición de la mayoría de los consumidores a la variabilidad de precios por pagar tarifas fijas, provoca moderadas reacciones del consumo ante incrementos significativos del precio. Además, la restricción de la capacidad disponible en los periodos de demanda punta también dificulta la reacción de la oferta ante comportamientos unilaterales de subida de precios. Adicionalmente, las estructuras de mercado altamente concentradas heredadas del marco previo a la liberalización podrían favorecer la emergencia de un equilibrio colusivo, aunque sea tácito. Todo esto aconseja la introducción de fuertes reformas estructurales y una continua supervisión del comportamiento de los mercados mayoristas de electricidad para garantizar un funcionamiento lo más competitivo posible.

Pero, las empresas eléctricas también pueden abusar de su poder de mercado sin necesidad de alterar el funcionamiento competitivo del mercado mayorista. Realizando estratégicamente bajos niveles de inversión en nueva capacidad pueden elevar el número de horas en las que la capacidad está restringida provocando fuertes subi-

${ }^{12}$ En un análisis sencillo de monopolio con demanda y costes lineales se puede demostrar que el coste de un mecanismo similar al analizado para incentivar el nivel de inversión óptimo sería mayor que el máximo bienestar social alcanzable. Con un oligopolio el coste sería algo menor pero también muy importante. 
das del precio mayorista. Además, por las características técnicas de la industria eléctrica, la falta de capacidad de generación puede exigir la intervención del operador del sistema para restaurar el equilibrio entre oferta y demanda a través del racionamiento aleatorio, todo ello en detrimento del bienestar social.

En este trabajo se estudia el alcance del efecto del poder de mercado sobre el nivel de inversión en capacidad de generación de electricidad. Mediante simulaciones con datos del sistema eléctrico español se observa que la reducción de la inversión por parte de las empresas eléctricas puede llegar a casi el 20\% de la demanda máxima para un escenario con un número reducido de empresas que ofertan su energía a precios competitivos. Esto representaría un déficit de casi un $40 \%$ respecto del nivel de capacidad que se considera necesario para alcanzar un nivel de garantía de suministro razonable.

Además, se muestra que los mecanismos de incentivos basados en el precio similares a los aplicados en el caso español no son eficaces por presentar un perfil inadecuado, y resultar excesivamente costosos. Por otra parte, este tipo de mecanismos adolece de otras debilidades. Al igual que con el mecanismo de "price adder" del pool británico en sus inicios, que consiste en añadir un suplemento al precio final basado en la probabilidad de falta de suministro, a las empresas eléctricas no les interesa alcanzar el nivel óptimo de capacidad para evitar que la remuneración por capacidad sea nula. Por otro lado, para alcanzar el nivel de capacidad deseado se exige una gran coordinación entre empresas que no resulta fácil en la práctica.

\section{Referencias bibliográficas}

[1] AURIOL, E. y BIANCINI, S. (2009): "Economic integration and investment incentives in regulated industries", CEPR Disussion Paper No. 7296.

[2] CASTRO-RODRÍGUEZ, F., MARÍN, P. L. y SIOTIS, G. (2009): "Capacity choices in liberalised electricity markets", Energy Policy 37, pp. 2574-2581.

[3] CNE (2009): "Informe marco sobre la demanda de energía eléctrica y gas natural, y su cobertura", Comisión Nacional de Energía (CNE), 22 de diciembre de 2009.

[4] CRAMTON, P. y STOFT, S. (2006): "The Convergence of market designs for adequate generating capacity with special attention to the CAISO's resource aequacy problem", Manuscript, 25 April. (disponible en http://stoft.com/metaPage/lib/Cramton-Stoft-EOB2006-04-ICAP-energy-convergence.pdf)

[5] DE VRIES, L. J. (2007): "Generating adequacy: Helping the market do this job", Utilities Policy 15, pp. 20-35.

[6] FEDERICO, G. y VIVES, X. (2008): "Competition and regulation in the Spanish gas and electricity markets", IESE Business School, University of Navarra. (con la colaboración de N. Fabra).

[7] FABRA, N. (2007): "La Electricidad: mercados, inversiones, y garantía de suministro", Economía Industrial, $\mathrm{N}^{\circ} 364$, pp. 63-74.

[8] FABRA, N.; VON DER FEHR, N-H. y DE FRUTOS, M. A. (2008): "Investment incentives and auction design in electricity markets", CEPR Discusión Papers No. 6626. 
[9] GARCÍA, A. y STACCHETTI, E. (2009): “Investment dynamics in electricity markets”, Economic Theory, DOI 10.1007/s00199-009-0508-3.

[10] GRIMM, V. y ZOETTL, G. (2006): "Capacity choice under uncertainty: the impact of market structure", Working Paper Series in Economics, University of Cologne. (disponible en http://www.wiso.uni-koeln.de)

[11] JOSKOW, P. L. (2007): “Competitive electricity markets and investment in the new generating capacity", in Helm, D. (Ed.), The New Energy Paradigm. Oxford University Press.

[12] JOSKOW, P. L. (2008): “Capacity payment in imperfect electricity markets: Need and design”, Utilities Policy 16, pp. 159-170.

[13] NEUHOFF, K. y DE VRIES, L. (2004): "Insufficient incentives for investment in electricity generations", Utilities Policy 12, pp. 253-267. 
